\title{
Screening of differentially expressed genes in the growth plate of broiler chickens with Tibial Dyschondroplasia by microarray analysis
}

Wen-xia Tian ${ }^{1,2,3}$, Jia-kui Li ${ }^{1}$, Ping Qin ${ }^{1}$, Rui Wang ${ }^{1}$, Guan-bao Ning ${ }^{2}$, Jian-gang Qiao ${ }^{2}$, Hong-quan Li ${ }^{2}$, Ding-ren Bi ${ }^{4}$, Si-yi Pan ${ }^{3}$ and Ding-zong Guo ${ }^{1^{*}}$

\begin{abstract}
Background: Tibial dyschondroplasia (TD) is a common skeletal disorder in broiler chickens. It is characterized by the presence of a non-vascularized and unmineralized cartilage in the growth plate. Previous studies have investigated differential expression of genes related to cartilage development during latter stages of TD. The aim of our study was to identify differentially expressed genes (DEGs) in the growth plate of broiler chickens, which were associated with early stage TD. We induced TD using tetramethylthiuram disulfide (thiram) for 1, 2, and 6 days and determined DEGs with chicken Affymetrix GeneChip assays. The identified DEGs were verified by quantitative polymerase chain reaction (qPCR) assays.
\end{abstract}

Results: We identified 1630 DEGs, with 82, 1385, and 429 exhibiting at least 2.0-fold changes $(P<0.05)$ at days 1, 2 , and 6 , respectively. These DEGs participate in a variety of biological processes, including cytokine production, oxidation reduction, and cell surface receptor linked signal transduction on day 1 ; lipid biosynthesis, regulation of growth, cell cycle, positive and negative gene regulation, transcription and transcription regulation, and antiapoptosis on day 2; and regulation of cell proliferation, transcription, dephosphorylation, catabolism, proteolysis, and immune responses on day 6 . The identified DEGs were associated with the following pathways: neuroactive ligand-receptor interaction on day 1 ; synthesis and degradation of ketone bodies, terpenoid backbone biosynthesis, ether lipid metabolism, JAK-STAT, GnRH signaling pathway, ubiquitin mediated proteolysis, TGF- $\beta$ signaling, focal adhesion, and Wnt signaling on day 2; and arachidonic acid metabolism, mitogen-activated protein kinase (MAPK) signaling, JAK-STAT, insulin signaling, and glycolysis on day 6 . We validated seven DEGs by qPCR.

Conclusions: Our findings demonstrate previously unrecognized changes in gene transcription associated with early stage TD. The DEGs we identified by microarray analysis will be used in future studies to clarify the molecular pathogenic mechanisms of TD. From these findings, potential pathways involved in early stage TD warrant further investigation.

\section{Background}

Tibial dyschondroplasia (TD) is a common skeletal disorder in broiler chickens characterized by the presence of non-vascularized and unmineralized cartilage in the growth plate. Tetramethylthiuram disulfide (thiram) exerts its cytotoxic effects through membrane damage, mitochondrial injury, inhibition of glutathione metabolism, cell death, and inhibition of angiogenesis. Rath

\footnotetext{
*Correspondence: hlgdz@163.com

${ }^{1}$ College of Veterinary Medicine, Huazhong Agricultural University, Wuhan 430070, China

Full list of author information is available at the end of the article
}

et al. [1] showed that thiram-induced TD is not produced through an increase in chondrocyte multiplication in the transition zone, nor by altering the expression of genes causing the arrest of chondrocytes in a pre-hypertrophic state. It acts by creating metabolic dysfunction that leads to the destruction of blood capillaries in the transition zone chondrocytes. Our previous studies showed that thiram could promote chondrocyte proliferation in the growth plate of chickens, and disturbs the regulation of endochondral calcification and development of normal cartilage. This results in prehypertrophic cell accumulation, angionecrosis, abnormal extracellular

\section{Biomed Central}




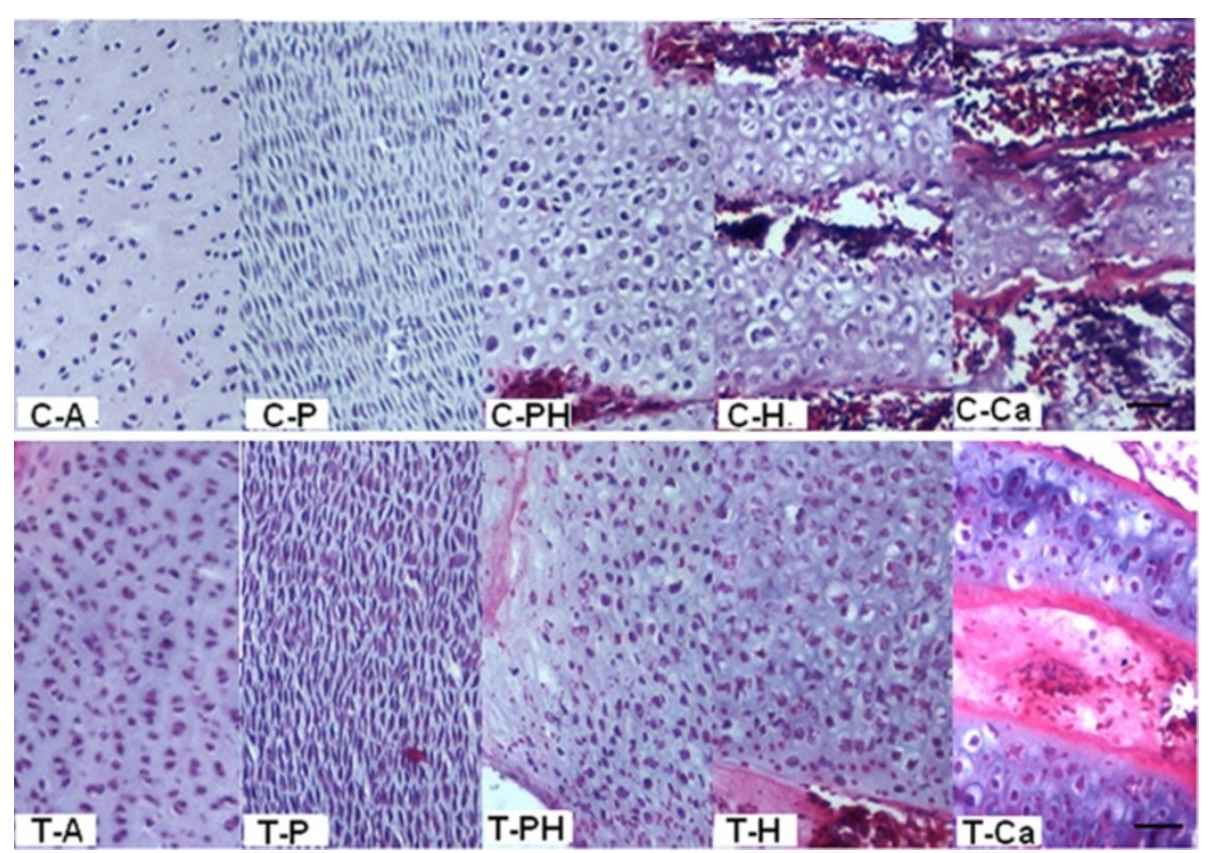

Figure 1 Histologic characteristics of tibia growth plate development in broiler chickens at day 4 during the experimental period. C, control; T, thiram-fed; A, articulus zone; P, proliferative zone; PH, pre-hypertrophic zone; $\mathrm{H}$, hypertrophic zone; Ca, calcification zone. Thiram promoted chondrocyte proliferation, resulting in necrosis of a large number of cells, and compressed blood vessels that led to necrosis of blood vessels. The scale bar indicates $200 \mu \mathrm{m}$.

matrix synthesis, deferred endochondral calcification, and bone resorption (Figure 1) [2].

Previous studies have investigated the differential expression of genes related to cartilage development during late stage TD using various techniques, such as immunohistochemistry, western blotting, in situ hybridization, semiquantitative polymerase chain reaction (PCR), real-time quantitative PCR (qPCR), and proteomic analyses. These studies revealed abnormal expression of a variety of factors during TD, including extracellular matrix molecules (collagen, osteopontin, osteonectin), growth factors, and hormones [vascular endothelial growth factor (VEGF) and its receptor, hypoxia inducible factor-1 (HIF-1)]. Additionally, matrix metalloproteinases (MMPs), carbonic anhydrase II (CA2), and heat shock proteins (HSPs) were also shown to be affected [3-11]. Recently we generated cDNA libraries for 96-h thiram-induced broiler chicken TD using a suppression subtractive hybridization (SSH) technique, and identified 10 differentially expressed genes (DEGs). Five of these DEGs were found to be upregulated in chicken TD: matrilin-3 (matn3); chondromodulin-I (chm-1); NADH dehydrogenase (nadhdh); cytochrome C oxidase subunit III (coxIII); and enolase 1 (eno1). Differential expression of these genes may affect cartilage matrix cross-linking, angiogenesis, energy metabolism, and growth regulation [12]. The average cycle of chondrocyte proliferation to terminal differentiation is approximately $21 \mathrm{~h}$; with thiram-induced cytotoxicity accompanied by rapid and significant oxidation, lipid peroxidation, and cell death. Thus, it is likely that the changes that occur during the early stages of TD initiate alterations that are found during late stage TD.

The use of DNA microarray techniques has made it possible for large-scale analysis of gene function and regulation to be conducted. DNA microarrays have been used to study skeletal development in humans and rodents [13-15]. In this study, we screened temporal changes in DEGs during early stages of TD in broiler chickens using an Affymetrix GeneChip, and verified expression patterns at different phases by qPCR. Genetic analyses were used to complement morphological changes and better understand the pathogenic mechanisms of TD.

\section{Results}

\section{Pathological features of thiram-induced TD}

Pathologic characteristics of broiler chicken tibia growth plate induced by thiram at day 1 were ascertained (Figure 2). Macroscopic examination revealed that the growth plates of chickens administered thiram were thickened compared with the control group (Figure 2a). Histological characteristics of the growth plates of these chickens were observed by microscopy, and revealed that chondrocytes at the thickened proliferative and pre-hypertrophic zones were sparse. Pre-hypertrophic and hypertrophic chondrocytes exhibited pyknosis. Additionally, the number of empty cartilage capsules was increased and the number of blood vessels 


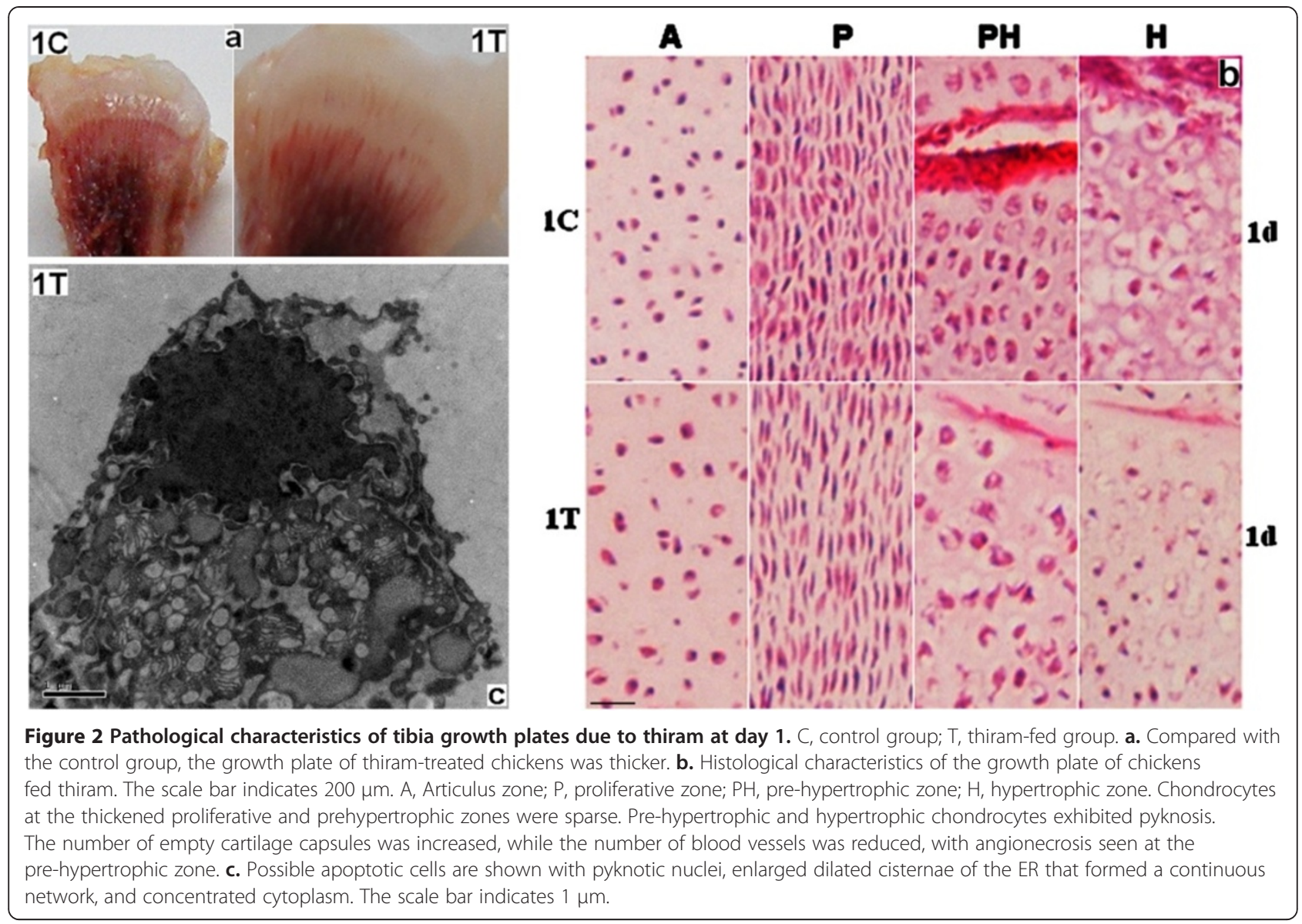

decreased, with angionecrosis observed at the prehypertrophic zone (Figure 2b). Transmission electron microscopy was used to examine apoptotic cells that contained pyknotic nuclei. These cells exhibited dilated cisternae of the endoplasmic reticulum (ER) that were enlarged and formed a continuous network, with a concentrated cytoplasm (Figure 2c).

\section{Isolation and assessment of total RNA}

Total RNA was isolated from the growth plates of control and thiram-treated chickens on days 1, 2 or 6 postfeeding. Total RNA quality was determined sufficient for subsequent analyses when RNA integrity was 2 (distinct bands corresponding $28 \mathrm{~S}$ and $18 \mathrm{~S}$ rRNA at a ratio of 2:1), total RNA concentration was between $2-4 \mu \mathrm{g} / \mu \mathrm{L}$, the $A_{260 / 280}$ ratio was around 2.1 , and the $A_{260 / 230}$ ratio of a sample was near 2.2.

\section{DEGs at different stages of TD}

Our one-way ANOVA revealed 1630 DEGs with a minimum 2.0-fold change $(p<0.05)$ in expression level. There were 82,1385 , and 429 DEGs identified on days 1,2 , and 6, respectively (Additional file 1). On day 1, 61 DEGs were upregulated and the remaining 21 downregulated. On day 2, 641 DEGs were upregulated and 744 downregulated, while on day 6, 324 DEGs were upregulated and 105 downregulated. Only 29 DEGs were commonly observed at all three time points; 18 DEGs were observed for both days 1 and 2; 13 DEGs were observed on days 1 and 6; and 177 DEGs were commonly observed for days 2 and 6 (Figure 3). And there were 39, 1086, and 279 DEGs (2.0-fold change, $p<0.05$ ) identified on days 1, 2, and 6 by two-way ANOVA (Additional file 2).

\section{Reliability of microarray screening}

Microarray screening assays revealed 18 hybridization maps. All maps showed a regular dot array with excellent signal saturation and homogeneous background. Quality control reports also indicated a stable background around 30, and a noise level of $1.14 \%$. At the experimental setting $(\alpha 1=0.04, \alpha 2=0.06, \tau=0.015)$, the marginal signal intensity $(\mathrm{M})$ was approximately $2.2 \%$, thereby confirming the reliability of the microarrays.

To further validate the microarrays, seven up-regulated genes (1.3-73.5-fold) were selected for qPCR analysis. Of these genes, heat shock protein 25 (hsp25), and lysyl 


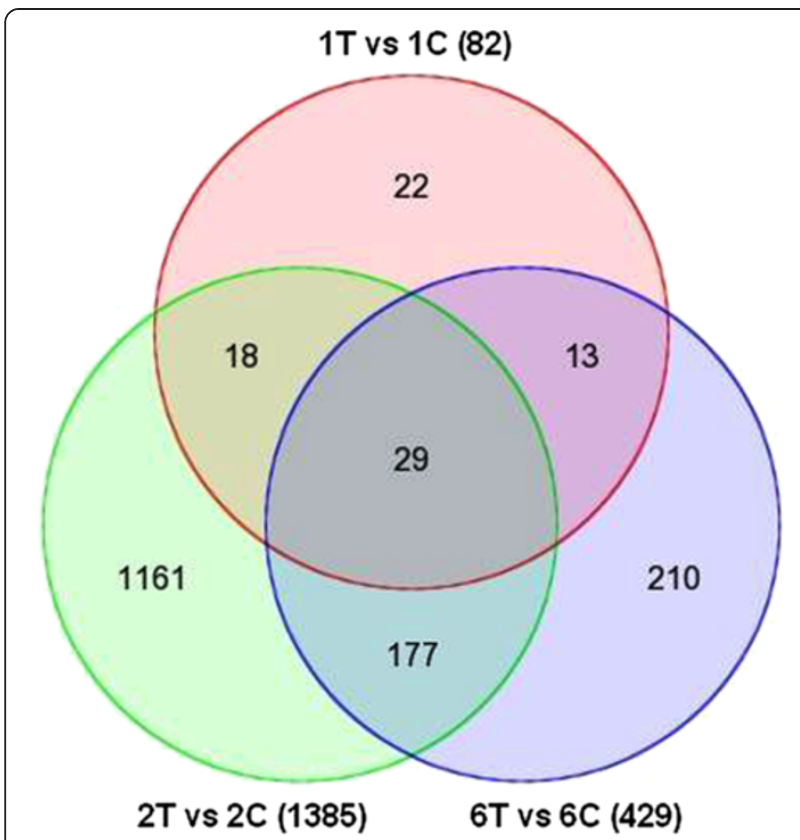

Figure 3 Analysis of the 1630 transcripts identified on days 1,2 and 6 using Venn diagrams. 1C, 2C, and $6 C$ indicates days 1,2 and 6 for the control group, respectively, while 1T, 2T, and $6 T$ denotes days 1,2 , and 6 for the thiram-fed group, respectively. There were 82, 1385, and 429 DEGs identified on days 1, 2, and 6, respectively. Only 29 DEGs were observed at all three time points. Thirteen DEGs were observed on both days 1 and 6; 18 DEGs were observed on both days 1 and 2; 177 DEGs were observed on both days 2 and 6 .

oxidase (lox) expression were significantly upregulated $(p<0.05)$ at days 1,2 and 6 . However, kinectin $1(k t n 1)$, inhibitor of DNA binding 1 (id-1), secreted frizzledrelated protein $4(s f r p 4)$, cadherin $1(c d h 1)$, and enolase 2 (eno2) showed significant $(p<0.05)$ differential expression at two time points. Despite consistent trends of differential expression, the qPCR results did not agree with the microarray data with respect to the range in fold-change (FC) range (Table 1).

\section{Cluster analyses of DEGs}

Principal component analysis (PCA) revealed a similarity of $37.7 \%$ at the three time points examined. There was a relatively minor difference in DEGs observed between control and thiram-fed chickens at day 1 . However, the differences in DEGs between the two groups were significantly different at days 2 and 6 (Figure 4). A clear expression pattern emerged after hierarchical clustering analyses of the 1630 transcripts $(p<0.05, \mathrm{FC} \geq 2.0)$ on days 1,2 , and 6. Hierarchical cluster analysis also showed that chickens in the control group on days 1,2 , and 6 formed a cluster with similar gene expression patterns. Gene expression patterns of thiram-fed chickens on days 1 and 6 were more similar to those observed in control animals. The gene expression patterns in thiramfed chickens at day 2 formed a separate cluster with similar gene expression patterns (Figure 5).

\section{DEGs gene ontology (GO) analyses}

Annotation of identified DEGs was carried out using the Database for Annotation, Visualization and Integrated Discovery (DAVID) at the three time points examined. These DEGs were found to participate in a variety of biological processes, such as cytokine production $(p<0.05)$, cell adhesion, intracellular signaling cascades, cell surface receptor linked signal transduction, oxidation reduction and phosphate metabolic processes on day 1 (Figure 6). On day 2 DEGs were associated with transcription regulation, sterol metabolic processes, lipid biosynthetic processes, growth regulation, steroid metabolism, regulation of cell morphogenesis, the mitotic cell cycle, fatty acid metabolism, cellular amino acid derivative metabolism, anti-apoptosis, the cell cycle, positive and negative gene regulation $(p<0.05)$ (Figure 7). On day 6, DEGs were associated with positive regulation of the developmental process, transcription, immune responses, bone mineralization and positive regulation of cell differentiation $(p<0.01)$, proteolysis, protein amino acid dephosphorylation, negative regulation of signal transduction, regulation

Table 1 Validation of microarray data by qPCR

\begin{tabular}{|c|c|c|c|c|c|c|c|}
\hline \multirow[t]{2}{*}{ Gene } & \multirow[t]{2}{*}{ Gene description } & \multicolumn{3}{|c|}{ Microarray FC } & \multicolumn{3}{|c|}{ qPCR FC } \\
\hline & & d 1 & d 2 & d 6 & d1 & d2 & d6 \\
\hline lox & lysyl oxidase & +3.9 & +71.8 & +9.6 & +4.4 & +212.9 & +13.8 \\
\hline hsp25 & heat shock protein 25 & +2.1 & +43.3 & +25.1 & +3.4 & +298.0 & +30.5 \\
\hline id1 & inhibitor of DNA binding 1 & $+1.6^{a}$ & +2.9 & +2.3 & $+1.1^{\mathrm{a}}$ & +1.8 & $+1.2^{a}$ \\
\hline$k \operatorname{tn} 1$ & kinectin 1 & $+1.5^{\mathrm{a}}$ & +4.7 & +3.6 & $+1.9^{a}$ & +17.1 & +5.7 \\
\hline sfrp4 & secreted frizzled-related protein 4 & +2.8 & +73.5 & +11.6 & $+2.0^{a}$ & +52.2 & +11.2 \\
\hline cdhl & cadherin 1 & $+1.3^{\mathrm{a}}$ & +31.4 & +10.9 & $+2.4^{a}$ & +45.4 & +3.1 \\
\hline eno2 & enolase 2 & +2.3 & +10.5 & +4.0 & +5.1 & +6.2 & $+1.1^{\mathrm{a}}$ \\
\hline
\end{tabular}

+ Represents upregulated. ${ }^{a}$ Represents $p>0.05 ; \mathrm{d} 1, \mathrm{~d} 2$, and $\mathrm{d} 6$ denote the three time points of analysis. 


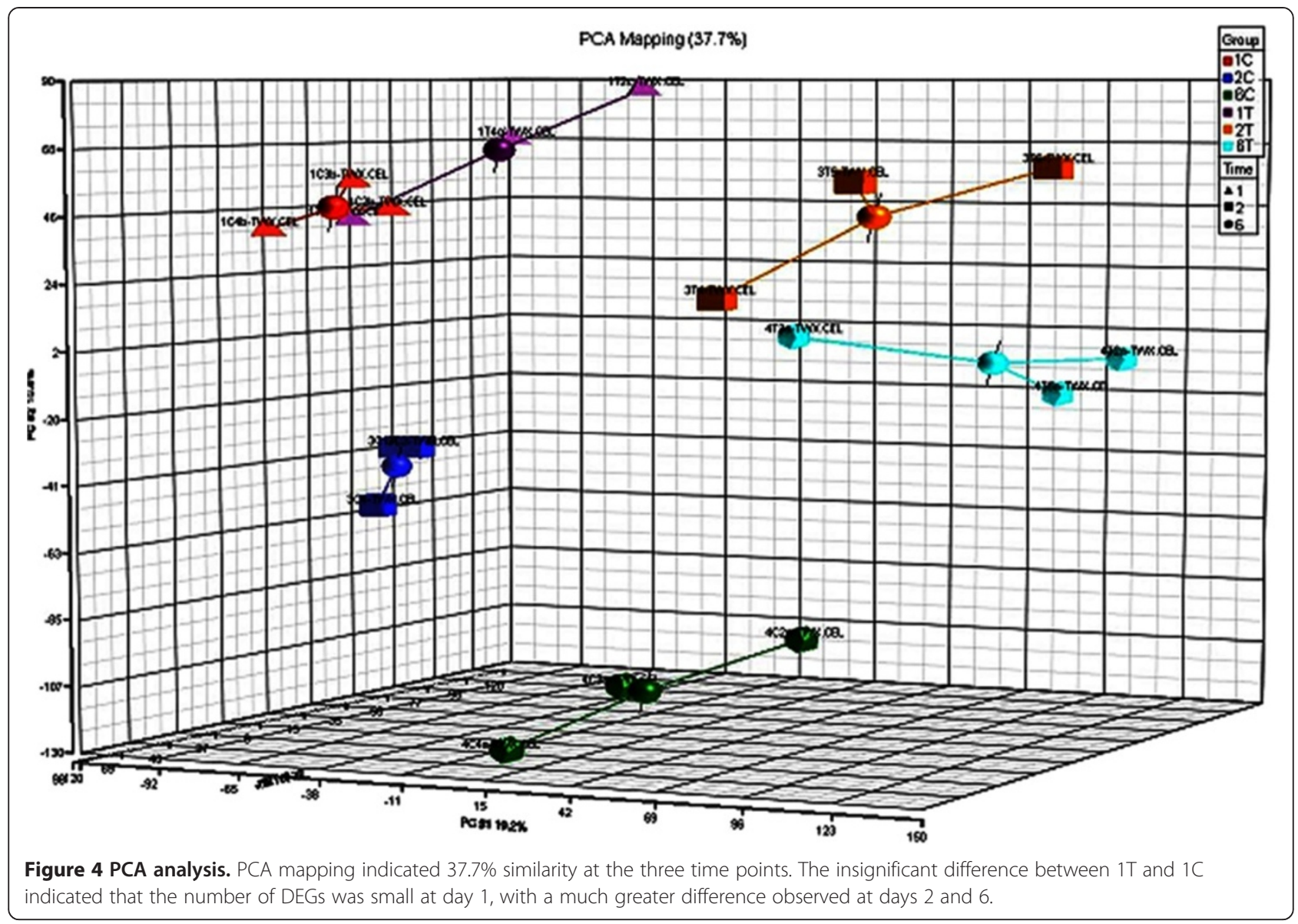

of cell proliferation, negative regulation of cell communication, and catabolic processes $(p<0.05)$ (Figure 8 ).

\section{Potential metabolic pathways related to TD}

A number of metabolic pathways were possibly related to TD development between days 1 and 6. Pathways associated with DEGs were: neuroactive ligand-receptor interaction $(p<0.05)$; the phosphatidylinositol signaling system; calcium signaling pathways; and the MAPK signaling pathway on day 1 . Synthesis and degradation of ketone bodies, ether lipid metabolism, terpenoid backbone biosynthesis, JAK-STAT, and steroid biosynthesis $(p<0.05)$, GnRH signaling pathway, ubiquitin mediated proteolysis, TGF- $\beta$ signaling, MAPK signaling, VEGF signaling, focal adhesion, Wnt signaling, and regulation of the actin cytoskeleton were all pathways associated with DEGs identified on day 2. Arachidonic acid metabolism $(p<0.01)$, the MAPK signaling pathway $(p<0.05)$, JAK-STAT, glycolysis, Adipocytokine signaling pathway, insulin signaling, cytokine-cytokine receptor interaction, TGF- $\beta$ signaling, focal adhesion and regulation of the actin cytoskeleton were associated with DEGs on day 6. Of these DEGs, the expression level of secreted frizzled-related protein $4(s f r p 4)$ was upregulated 2.8-, 73.5-, and 11.6-fold on days 1, 2, and 6 , respectively. The expression level of cadherin 1 (cdh1) was upregulated 31.4- and 10.9-fold on days 2 and 6, respectively. Similarly, expression of enolase 2 (eno2) was upregulated 2.3-, 10.5-, and 4.0-fold on days 1,2 , and 6 , respectively.

\section{Discussion}

Previous studies of TD have focused on the late stages; however, in this study we have reported the pathological changes of thiram-induced TD at earlier stages. Our findings show that the growth plate in chickens given thiram was thickened, with a decreased number of chondrocytes at the thickened proliferative and pre-hypertrophic zone. The pre-hypertrophic and hypertrophic chondrocytes exhibited pyknosis. We also observed an increased number of empty cartilage capsules and a decreased number of blood vessels, in addition to angionecrosis at the prehypertrophic zone. Transmission electron microscopy analysis of apoptotic cells revealed pyknotic nuclei, and dilated cisternae of the ER that were enlarged and formed a network structure; also, the cytoplasm was concentrated.

Biological process annotation revealed that the DEGs at day 1 following feeding with the thiram diet were 


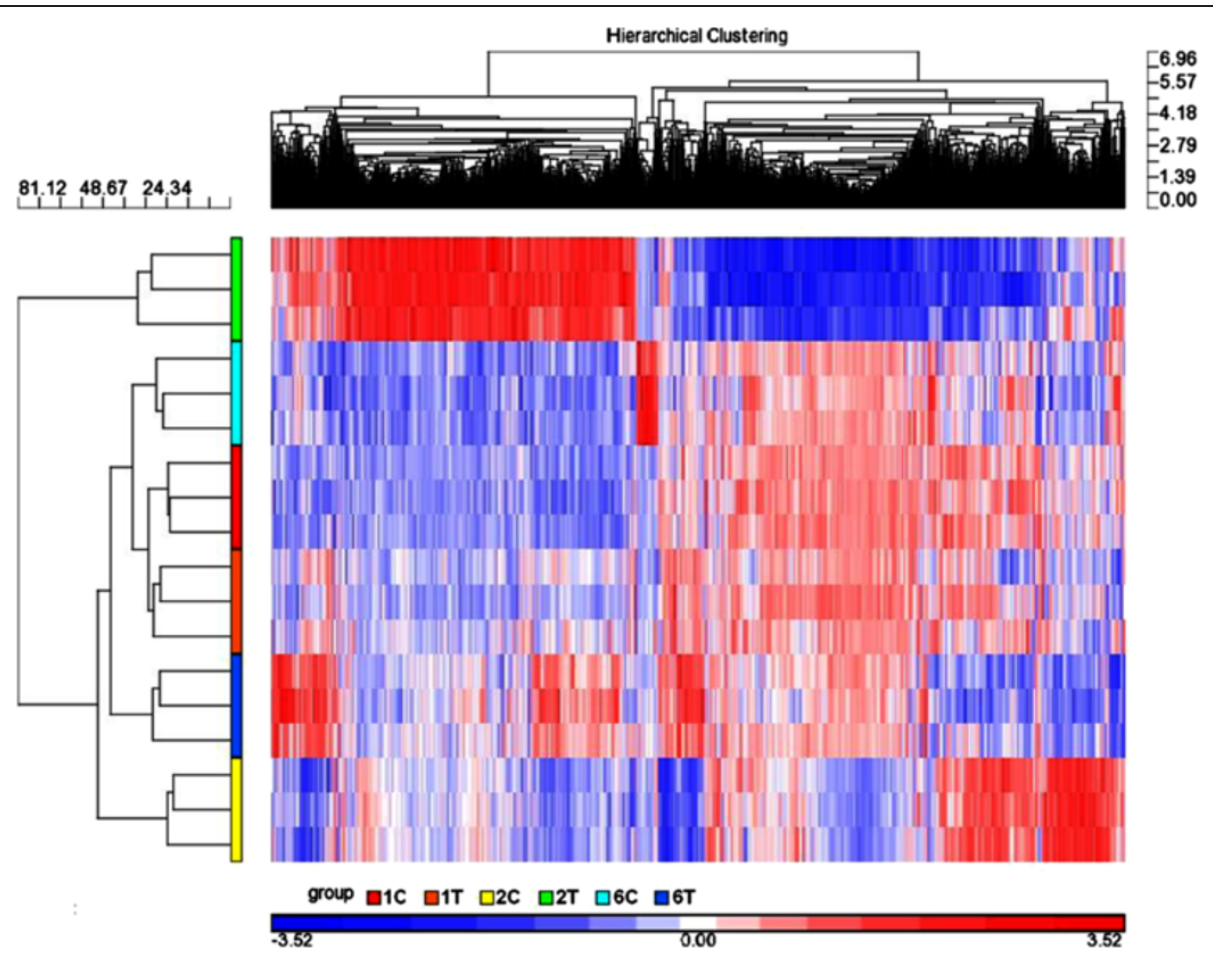

Figure 5 Hierarchical clustering analysis of differentially expressed genes during TD. Hierarchical clustering analyses of the 1630 transcripts $(p<0.05, F C \geq 2.0)$ on days 1,2 , and 6 . Red, gray, and blue represent high, average, and absent expression levels of genes, respectively. Each row represents one chicken, and each column refers to a gene. $1 C, 2 C$, and 6 indicates days 1,2 and 6 for the control group respectively, while $1 T$, $2 T$, and 6 T denotes days 1, 2, and 6 for the thiram-fed group, respectively.

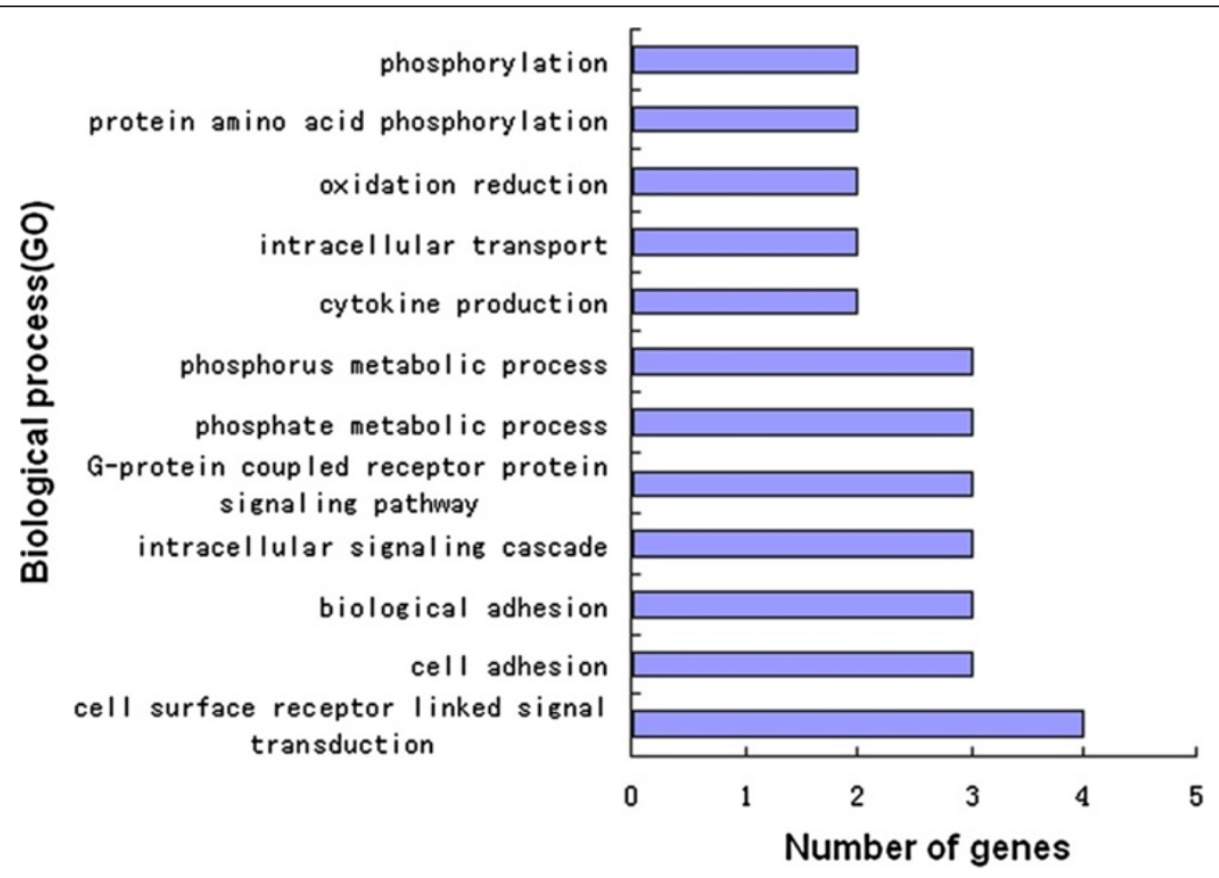

Figure 6 Biological process Gene Ontology (GO) analysis of 82 DEGs on day 1. 


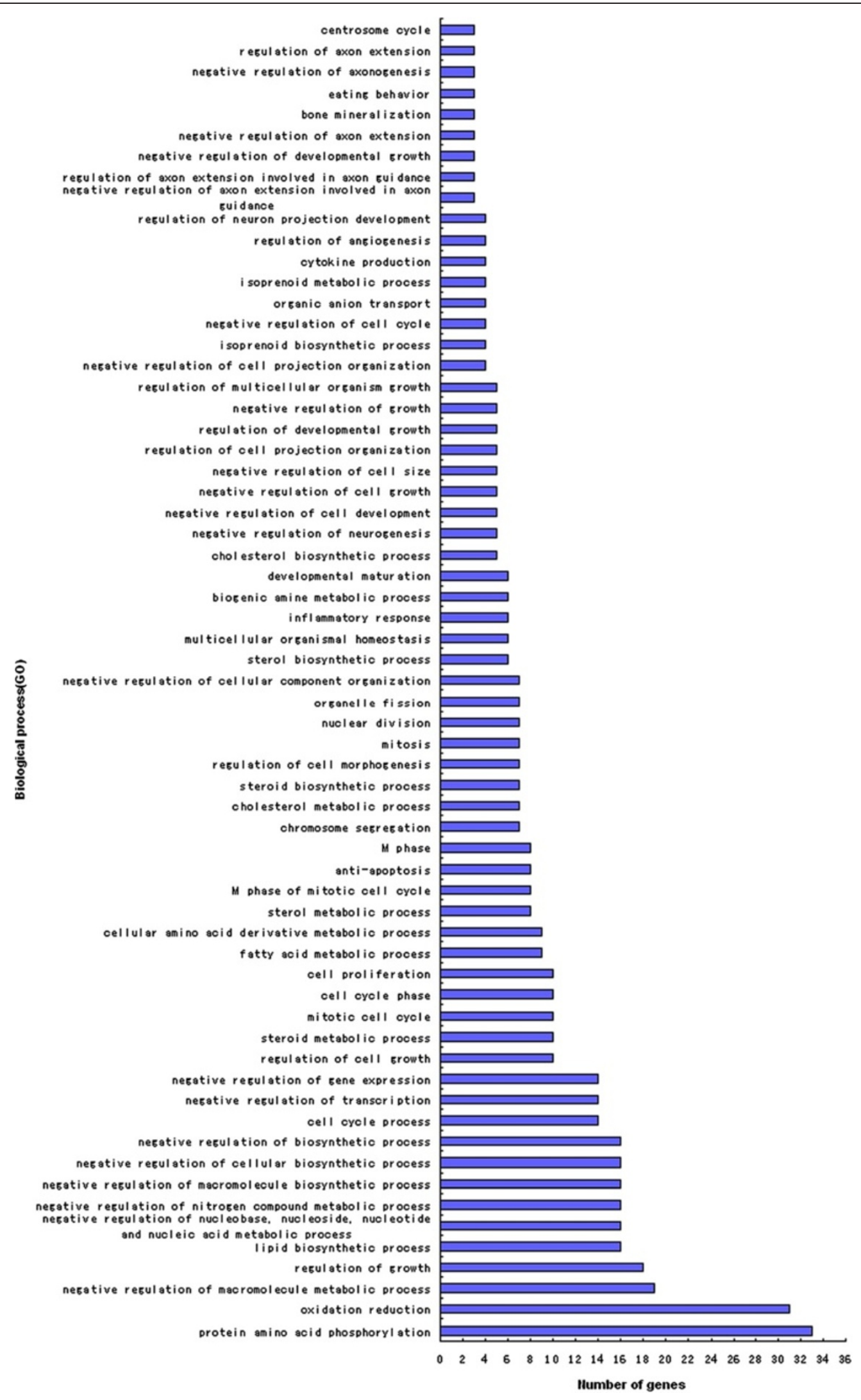

Figure 7 Biological process Gene Ontology (GO) analysis of 1385 DEGs on day 2. 


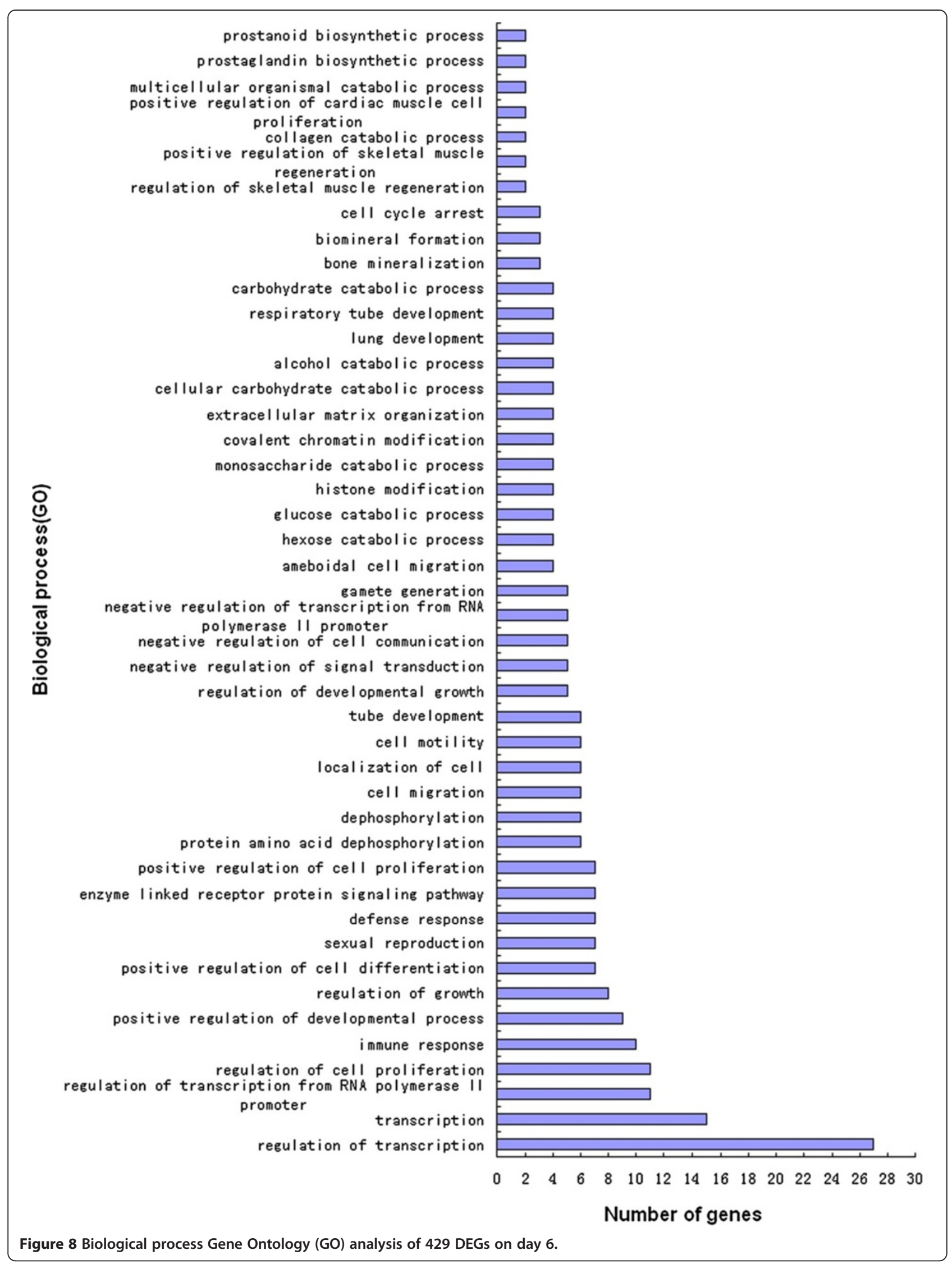


associated with cytokine production that primarily involved the neuroactive ligand-receptor interaction pathway. The downregulated genes included chemokine $(\mathrm{C}-\mathrm{C}$ motif) receptor 6 (ccr6), prostaglandin $\mathrm{E}$ receptor 4 (subtype EP4) (ptger4), arginine vasopressin receptor 2 (avpr2), and proprotein convertase subtilisin/kexin type 5 (pcsk5), while 5-hydroxytryptamine (serotonin) receptor 7 (htr7), and opiate receptor-like 1(oprl1) were upregulated.

Genes screened on day 2 revealed the involvement of important biological processes such as sterol metabolism, lipid biosynthesis, steroid metabolism, and terpenoid backbone biosynthesis in particular. Terpenoid, also called isoprenoid, plays an important role during cell growth. Ether lipids, also called plasmalogens or 1-O-alkyl lipids, are ubiquitous and sometimes form major parts of cell membranes in mammals. Differences between the catabolism of ether glycerophospholipids by specific phospholipases might be involved in the generation of lipid second messenger systems, which are important in signal transduction. Ether lipids can also act directly in cell signaling, as the platelet-activating factor is an ether lipid signaling molecule that is involved in leukocyte function in mammalian immune systems. Another possible function of plasmalogen ether lipids are as antioxidants to protect against oxidative stress, therefore these lipids might play a role in serum lipoprotein metabolism. This antioxidant activity comes from the enol ether double bond that is targeted by a variety of reactive oxygen species [16]. Sterols are natural active compounds that form major parts of the cell membranes and are precursors of hormones, vitamin D and sterol compound. Thus, downregulation in the expression of synthases is likely to contribute to the progression of TD.

Analysis of gene expression on day 6 revealed that the biological processes affected were associated with positive regulation of developmental processes involved in arachidonic acid metabolism and the MAPK signaling pathway. Arachidonic acid is the direct precursor of active compounds such as prostaglandin E2, prostaglandin I2 and leukotrienes C4 (LTC4). These play important roles in the regulation of lipoprotein metabolism, cell development, blood vessel elasticity and white blood cell function.

From these GO annotations at all time points, we deduced that the cytotoxic effects of thiram resulted in membrane damage, loss of enzymatic activities, and loss of protein receptor function, especially cysteine-rich proteins. This is likely to influence signal transduction, thereby leading to cell apoptosis and inhibition of pathological angiogenesis.

Our analyses also suggest that thiram-induced TD at the early stages is primarily associated with multiple metabolic pathways, focal adhesion, Jak-STAT, adipocytokine signaling pathway, GnRH signaling pathway, oxidative phosphorylation, glycolysis, Wnt, TGF- $\beta$, VEGF, and insulin signaling pathways. Metabolic pathways associated with DEGs at days 2 and 6 were identical. Impaired mitochondrial energy metabolism occurred during the initial stages of TD, with glycolysis subsequently induced at later stages of TD. The focal adhesion and JAK-STAT signaling pathways account for most DEGs, where focal adhesion and JAK-STAT may promote chondrocyte proliferation, development, antiapoptosis, cell survival, and cell motility through Wnt or MAPK signaling pathways. During early stages of TD, many genes were found to have altered expression, due to the cytotoxic effects of thiram, therefore the proliferation of growth plate chondrocytes and metabolism were altered.

Horvat-Gordon et al. [17] studied gene expression in chondrocytes isolated from the proliferative and hypertrophic zones of the avian growth plate using microarrays. They identified a number of genes associated with chondrocyte hypertrophy. Our preliminary analyses of the seven verified DEGs revealed that lysyl oxidase (lox), which was upregulated, participates in crosslinking of extracellular collagen via oxidative deamination of lysine or hydroxylysine [18,19]. LOX also plays important roles in cell adhesion/proliferation/migration, the intracellular signal response, and malignant processes. Moreover, LOX reenters cells and aggregates around the nuclei after oxidative changes [20]. The LOX propeptide (LOX-PP) inhibits smooth muscle cell signaling and proliferation, thereby providing a feedback mechanism to inhibit pathological angiogenesis [21]. Heat shock protein 25 (Hsp25) acts as a molecular chaperone to stabilize proteins [22-24], maintain membrane integrity, protect nucleic acids, and stabilize the cytoskeleton [25]. Hsp25 is a marker of differentiated odontoblasts [26-29] and also participates in the MAPK signaling pathway [30]. Additionally, the roles of Hsp25 in osteoblast signaling have also been widely studied [31-34]. The ID-1 protein is a member of the ID protein family that has been implicated in the regulation of cellular differentiation, cell cycle progression, senescence, and apoptosis. Moreover, ID-1 also activates VEGF by enhancing the stability and activity of hypoxia-inducible factor-1a [35,36].

Kinectin 1 (KTN1) is a receptor protein in the reticular membrane. KTN1 mainly participates in intracellular vesicle transport and can also regulate protein synthesis in eukaryotic cells by anchoring the elongation factor- 1 complex onto the ER [37]. Tamamura et al. [38] suggested that $\mathrm{Wnt} / \beta$-catenin signaling regulates chondrocyte phenotype, maturation, and function during cartilage development. Such regulation is critical for growth plate formation, cartilage boundary definition, and endochondral ossification. Over-expression of SFRP4 may inhibit the proliferation of osteoblasts by antagonizing Wnt signaling [39]. SFRP4 may also cause CDH1 over-expression and thus affect cell adhesion and impair angiogenesis [40]. In addition, hypoxic conditions disrupt 
metabolism and ultimately results in ENO2 and ENO1 over-expression [12].

\section{Conclusion}

We identified global gene expression changes during early stages of thiram-induced TD. Downregulation of prostaglandin E receptor 4 (subtype EP4) (ptger4) and arginine vasopressin receptor 2 (avpr2) on day 1 directly influenced signal transduction and blood vessel elasticity. On day 2, downregulation of a variety of synthases altered the production of lipid compounds that are precursors of hormones, vitamin D, PTGD, and PTGE, all of which likely have an important role in angiogenesis, regulation of transcription and cell proliferation, and bone development. Taken together, the findings from this study contribute potential insights into therapies for TD, and also provide fundamental information regarding the pathological mechnisms involved during the early stages of TD.

\section{Methods}

\section{TD induction and tissue collection}

Broiler chickens (7 days old) were randomly divided into two groups. After fasting overnight, animals were fed a regular diet (control group) or a diet containing $100 \mathrm{mg} / \mathrm{kg}$ thiram for $48 \mathrm{~h}$ (thiram-fed group) to induce TD, as previously described [12]. Eight birds from each group were sacrificed by cervical dislocation under ether anesthesia on days 1,2 , and 6 after commencement of the experiment. The cartilage growth plates from individual birds were harvested, immediately frozen in liquid nitrogen, and stored at $-70^{\circ} \mathrm{C}$. All procedures were performed according to protocols approved by the Biological Studies Animal Care and Use Committee of Hubei Province, China.

\section{Gross and microscopic TD lesions}

On days 1, 2 and 6, tibiae were observed and recorded, and then immediately removed from two groups of chicks. Samples were fixed overnight in $4 \%$ paraformaldehyde or $2.5 \%$ glutaraldehyde in phosphate-buffered saline (PBS) at $4^{\circ} \mathrm{C}$. Serial histological sections (4- $\mu \mathrm{m}$ thickness) were prepared after samples had been fully decalcified in $10 \%$ ethylenediaminetetraacetic acid (EDTA) decalcifying fluid, dehydrated, embedded in paraffin wax, and then stained with hematoxylin and eosin. Sections were examined by light microscopy. For transmission electron microscopy, pre-fixed samples were post-fixed in $2 \% \mathrm{OsO} 4$, dehydrated, and embedded in epoxy resin. Ultrathin sections were stained with uranyl acetate and lead citrate, and observed with a HITACHI H-7650 transmission electron microscope at $80 \mathrm{kV}$ and a Gatan $832 \mathrm{CCD}$ camera.

\section{RNA extraction}

Individual cartilage growth plates collected from each group of birds were homogenized in TRIzol reagent to extract total RNA according to an improved method. RNA integrity and concentration were evaluated using denaturing formaldehyde gel electrophoresis and a Nanodrop 2000 analyzer (Thermo Scientific), respectively.

\section{Microarray hybridization and signal processing}

Total RNA samples from 18 chickens (three individual chickens from each group on days 1,2 and 6) were sent to GeneTech Biotech for hybridization to chicken

Table 2 Primers used for qPCR analysis

\begin{tabular}{|c|c|c|c|c|}
\hline Gene & Gene descriptions & Primers sequence $\left(5^{\prime}-3^{\prime}\right)$ & Target size (bp) & $\operatorname{Tm}\left({ }^{\circ} \mathrm{C}\right)$ \\
\hline \multirow[t]{2}{*}{$10 x$} & lysyl oxidase & Forward: TACGTGCAGAGGATGTCCATGT & 114 & 60 \\
\hline & & Reverse: TCTCAGGAGCACTCGGTTGTC & & \\
\hline \multirow[t]{2}{*}{ hsp25 } & heat shock protein 25 & Forward: AGGAGTGTGCCCATCCAGGT & 109 & 60 \\
\hline & & Reverse: GATGCAGACCGTTGTTCCGT & & \\
\hline \multirow[t]{2}{*}{ id1 } & inhibitor of DNA binding 1 & Forward: GGGGTCATTGCCGACATT A & 115 & 60 \\
\hline & & Reverse: AGGGGGGACTCAGGAATGTA & & \\
\hline \multirow[t]{2}{*}{ ktn1 } & kinectin 1 & Forward: CGGTGAATCTTAACCAGGATGTAG & 134 & 60 \\
\hline & & Reverse: TGCTATCCTCAGATCAGCGATT & & \\
\hline \multirow[t]{2}{*}{ sfrp4 } & secreted frizzled-related protein 4 & Forward: GCTGAATCTATCTGCTGTTGGG & 136 & 60 \\
\hline & & Reverse: CTAACACTGTAAGCATATTTCTGGC & & \\
\hline \multirow[t]{2}{*}{$c d h 1$} & cadherin 1 & Forward:TGAGAAGCAGATACTGAGCATTGTG & 122 & 60 \\
\hline & & Reverse: GCTCATCTTGGCCCCTTATCTC & & \\
\hline \multirow[t]{2}{*}{ eno2 } & enolase 2 & Forward: GTGTGCGTGTGTGTAGGTGTATGT & 113 & 60 \\
\hline & & Reverse: AGTGCTCAGAACGGAAGGAAGA & & \\
\hline \multirow[t]{2}{*}{ rps16 } & ribosomal protein S16 & Forward: ACAAACTGCTTGAACCTGTCCTC & 127 & 60 \\
\hline & & Reverse: GCTTTGGAAATAGCTTGACGG & & \\
\hline
\end{tabular}


Affymetrix GeneChips (Affymetrix). Briefly, total RNA (500 ng) was purified using a QIAGEN miRNeasy kit. The GeneChip IVT Labeling Kit (Affymetrix) was used for synthesis of biotin-labeled cRNA. Labeled cRNA $(15 \mu \mathrm{g})$ was hybridized to the GeneChip Chicken Genome Array at $45^{\circ} \mathrm{C}$ for $16 \mathrm{~h}$. GeneChips were washed and stained with a GeneChip Fluidics Station 450 (Affymetrix) using a standard protocol, and probe arrays were scanned using a Scanner 7G. Quality control (QC) data were obtained using Expression Console software. Principal component analysis (PCA) and histogram cluster analysis were conducted with Partek GS 6.4. Identification of DEGs was also conducted using Partek GS 6.4 through by one way ANOVA and two way ANOVA. The $p$-value cutoff for DEGs was set at 0.05 . The adjusted p-value was computed by the false discovery rate (FDR) using Partek GS 6.4, and FDR of approximately 5\% was set as a threshold. DEGs were subjected to hierarchical clustering using Cluster (version 3.0) and visualized with TreeView (version 1.6; Stanford University). The identified DEGs were analyzed for GO and biological pathways using DAVID (http://david.abcc.ncifcrf.gov).

\section{Verification of identified DEGs by qPCR}

Total RNA samples used for qPCR verification had also been used in the microarray analyses. Transcripts from each sample were amplified in triplicate and detected using a SYBR Green PCR Master Mix (Applied Biosystems). All primers used (Table 2) were synthesized by Genery Biotechnology. The RPS16 gene was used as the internal control for normalization, while the reference gene $\beta$-actin was used to verify the differences in expression levels. Data from qPCR assays were analyzed with Sequence Detector software (version 1.3.1; Applied Biosystems) and were performed by a variance analysis (ANOVA). A $P$-value less than 0.05 was considered significant.

\section{Additional files}

Additional file 1: We identified 1630 transcripts as being differentially expressed in the growth plate on days 1,2 and 6 with one way ANOVA. The $1 C, 2 C$, and 6 C designations refer to the control group on days 1,2 , and 6 , respectively. The $1 T, 2 T$, and $6 \mathrm{~T}$ designations refer to the thiram-fed group on days 1,2 and 6 , respectively. We have listed 82 transcripts in sheet 1T vs. 1C, 1385 transcripts in sheet 2T vs. 2C, and 429 transcripts in sheet 6T vs. 6C. A FC (gene expression level following administration of the thiram-containing diet compared with the control) greater than 2.0 represents upregulation, while an FC less than or equal to 2.0 represents downregulation. Ajusted $p$ value was computed by the false discovery rate (FDR) of approximately $5 \%$ using Partek GS 6.4.

Additional file 2: We identified 1222 transcripts as being differentially expressed in the growth plate on days 1, 2 and 6 with two way ANOVA. The $1 \mathrm{C}, 2 \mathrm{C}$, and $6 \mathrm{C}$ designations refer to the control group on days 1,2 , and 6 , respectively. The $1 T, 2 T$, and $6 \mathrm{~T}$ designations refer to the thiram-fed group on days 1,2 and 6, respectively. We have listed 39 transcripts in sheet 1T vs. 1C, 1086 transcripts in sheet 2T vs. 2C, and 279 transcripts in sheet 6T vs. 6C. A FC (gene expression level following administration of the thiram-containing diet compared with the control) greater than 2.0 represents upregulation, while an FC less than or equal to 2.0 represents downregulation. Ajusted $p$ value was computed by the false discovery rate (FDR) of approximately $5 \%$ using Partek GS 6.4.

\section{Competing interests}

The authors declare that they have no competing interests.

\section{Authors' contributions}

WX carried out the molecular genetic studies, sequence alignment, designed the study, performed the statistical analyses, and drafted the manuscript. P, R and JG extracted the total RNA. DR, SY, and DZ conceived the study, participated in its design and coordination, and helped draft the manuscript. JK, GB and HQ provided helpful discussion and advice. All authors read and approved the final manuscript.

\section{Acknowledgments}

This study was supported by grants from the China Postdoctoral Science Foundation (20080430984); the National Natural Science Foundation of China (31072179; 30571370); the Natural Science Foundation of Shanxi Province, China (2009011043-1); and Shanxi Agricultural University Scientific Research Foundation for Doctors. We thank Chang Shu and Dr An-dan Zhu for their assistance with the bioinformatics analysis.

\section{Author details}

${ }^{1}$ College of Veterinary Medicine, Huazhong Agricultural University, Wuhan 430070, China. ${ }^{2}$ College of Veterinary Medicine, Shanxi Agricultural University, Taigu 030801, China. ${ }^{3}$ College of Food Science and Technology, Huazhong Agricultural University, Wuhan 430070, China. ${ }^{4}$ State Key Laboratory of Agricultural Microbiology, College of Veterinary Medicine, Huazhong Agricultural University, Wuhan 430070, China.

Received: 29 April 2012 Accepted: 18 April 2013

Published: 23 April 2013

\section{References}

1. Rath NC, Richards MP, Huff WE: Changes in the tibial growth plates of chickens with thiram-induced dyschondroplasia. J Comp Path 2005, 133:41-52.

2. Tian WX, Li JK, Bi DR, Zhang YH, Qin P: Effect of thiram on growth performance and histopathologic changes of Tibial Dyschondroplasia in broiler chickens. Acta veterinaria et zootechnica Sinica 2008, 39:733-738.

3. Praul CA, Ford BC, Gay CV, Pines M, Leach RM: Gene expression and tibial dyschondroplasia. Poult Sci 2000, 79:1009-1013.

4. Webster SV, Farquharson C, Jefferies D, Kwan AP: Expression of type X collagen, Indian hedgehog and parathyroid hormone related-protein in normal and tibial dyschondroplastic chick growth plates. Avian Pathol 2003, 32:69-80.

5. Rath NC, Huff WE, Huff GR: Thiram-induced changes in the expression of genes relating to vascularization and tibial dyschondroplasia. Poult Sci 2007, 86:2390-2395.

6. Hasky-Negev M, Simsa S, Tong A, Genina O, Monsonego OE: Expression of matrix metalloproteinases during vascularization and ossification of normal and impaired avian growth plate. J Anim Sci 2008, 86:1306-1315.

7. Dan H, Simsa-Maziel S, Hisdai A, Sela-Donenfeld D, Monsonego OE: Expression of matrix metalloproteinases during impairment and recovery of the avian growth plate. J Anim Sci 2009, 87:3544-3555.

8. Genin O, Hasdai A, Shinder D, Pines M: Hypoxia, Hypoxia-Inducible Factor1 \{alpha\} (HIF-1\{alpha\}), and Heat-Shock Proteins in Tibial Dyschondroplasia. Poult Sci 2008, 87:1556-1564.

9. Velada I, Capela-Silva F, Reis F, Pires E, Egas C, Rodrigues-Santos P, Barros MT: Expression of genes encoding extracellular matrix macromolecules and metalloproteinases in avian tibial dyschondroplasia. J Comp Pathol 2011, 145:174-186.

10. Herzog A, Genin O, Hasdai A, Shinder D, Pines M: Hsp90 and angiogenesis in bone disorders - lessons from the avian growth plate. Am J Physiol Regul Integr Comp Physiol 2011, 301:R140-147. 
11. Rasaputra KS, Liyanage AB, Lay JO, Carthy FM, Rath NC: Tibial dyschondroplasia-associated proteomic changes in chicken growth plate cartilage. Avian Dis 2010, 54:1166-1171.

12. Tian WX, Zhang WP, Li JK, Bi DR, Guo DZ, Pan SY, Zhang YH, Qin P: Identification of differentially expressed genes in the growth plate of broiler chickens with thiram-induced tibial dyschondroplasia. Avian Pathol 2009, 38:160-166.

13. Wang Y, Middleton FA, Horton JA, Reichel L, Farnum CE, Damron TA: Microarray analysis of proliferative and hypertrophic growth plate zones identifies differentiation markers and signal pathways. Bone 2004 35:1273-1293.

14. James CG, Appleton CT, Ulici V, Underhill TM, Beier F: Microarray analyses of gene expression during chondrocyte differentiation identifies novel regulators of hypertrophy. Mol Bio Cell 2005, 16:5316-5333.

15. Grogan SP, Barbero A, Diaz-Romero J, Cleton-Jansen AM, Soeder S, Whiteside R, Hogendoorn PC, Farhadi J, Aigner T, Martin I, Mainil-Varlet P: Identification of markers to characterize and sort human articular chondrocytes with enhanced in vitro chondrogenic capacity. Arthritis Rheum 2007, 56:586-95.

16. Engelmann B: Plasmalogens: targets for oxidants and major lipophilic antioxidants. Biochem Soc Trans 2004, 32:147-150.

17. Horvat-Gordon M, Praul CA, Ramachandran R, Bartell PA, Leach RM Jr: Use of microarray analysis to study gene expression in the avian epiphyseal growth plate. Comp Biochem Physiol Part D Genomics Proteomics 2010, 5:12-23.

18. Lucero HA, Kagan HM: Lysyl oxidase: an oxidative enzyme and effector of cell function. Cell Mol Life Sci 2006, 63:2304-2016.

19. Liu PT, Stenger S, Li H, Wenzel L, Tan BH, Krutzik SR, Ochoa MT, Schauber J, Wu K, Meinken C, Kamen DL, Wagner M, Bals R, Steinmeyer A, Zügel U, Gallo RL, Eisenberg D, Hewison M, Hollis BW, Adams JS, Bloom BR, Modlin $\mathrm{RL}$ : Toll-like receptor triggering of a vitamin D-mediated human antimicrobial response. Science 2006, 311:1770-1773.

20. Kagan HM, Li W: Lysyl oxidase: properties, specificity, and biological roles inside and outside of the cell. J Cell Biochem 2003, 88:660-672.

21. Hurtado PA, Vora S, Sume SS, Yang D, Hilaire C, Guo Y, Palamakumbura AH, Schreiber BM, Ravid K, Trackman PC: Lysyl oxidase propeptide inhibits smooth muscle cell signaling and proliferation. Biochem Biophys Res Commun 2008, 366:156-161.

22. Gorman AM, Szegezdi E, Quigney DJ, Samali A: Hsp27 inhibits 6hydroxydopamine-induced cytochrome $\mathrm{C}$ release and apoptosis in PC12 cells. Biochem Biophys Res Commun 2005, 327:801-810.

23. Shimada M, Yamamoto M, Wakayama T, Iseki S, Amano O: Different expression of 25-kDa heat-shock protein (Hsp25) in Meckel's cartilage compared with other cartilages in the mouse. Anat Embryol (Berl) 2003 206:163-173.

24. Duverger O, Morange M: Heat shock protein 25 plays multiple roles during mouse skin development. Cell Stress Chaperones 2005, 10:268-277.

25. Du Y, Gu HJ, Gong QM, Yang F, Ling JQ: HSP25 affects the proliferation and differentiation of rat dental follicle cells. Int J Oral Sci 2009, 1:72-80.

26. Suzuki T, Nomura S, Maeda T, Ohsima H: An immunocytochemical study of pulpal responses to cavity preparation by laser ablation in rat molars by using antibodies to heat shock protein (Hsp) 25 and class II MHC antigen. Cell Tissue Res 2004, 315:311-319.

27. Kawagishi E, Nakakura-Ohshima K, Nomura S, Ohsima H: Pulpal responses to cavity preparation in aged rat molars. Cell Tissue Res 2006, 326:111-122.

28. Nakasone N, Yoshie $H$, Ohshima $H$ : An immunohistochemical study of the expression of heat-shock protein- 25 and cell proliferation in the dental pulp and enamel organ during odontogenesis in rat molars. Arch Oral Biol 2006, 51:378-386.

29. Nakasone $\mathrm{N}$, Yoshie $\mathrm{H}$, Ohshima $\mathrm{H}$ : The relationship between the termination of cell proliferation and expression of heat-shock protein-25 in the rat developing tooth germ. Eur J Oral Sci 2006, 114:302-309.

30. Thompson HS, Maynard EB, Morales ER, Scordilis SP: Exercise-induced HSP27, HSP70 and MAPK responses in human skeletal muscle. Acta Physiol Scand 2003, 178:61-72.

31. Kozawa O, Niwa M, Matsuno H, Ishisaki A, Kato K, Uematsu T: Stimulatory effect of basic fibroblast growth factor on induction of heat shock protein 27 in osteoblasts: role of protein kinase C. Arch Biochem Biophys 2001, 388:237-242.
32. Hatakeyama D, Kozawa O, Niwa M, Matsuno H, Ito H, Kato K: Upregulation by retinoic acid of transforming growth factor-beta-stimulated heat shock protein 27 induction in osteoblasts: involvement of mitogenactivated protein kinases. Biochim Biophys Acta 2002, 589:15-30.

33. Tokuda H, Kozawa O, Niwa M, Matsuno H, Kato K, Uematsu T: Mechanism of prostaglandin E2-stimulated heat shock protein 27 induction in osteoblast-like MC3T3-E1 cells. J Endocrinol 2002, 172:271-281.

34. Takai S, Tokuda H, Matsushima-Nishiwaki R, Hanai Y, Kato K, Kozawa O: Phosphatidylinositol 3-kinase/Akt plays a role in sphingosine 1-phosphate-stimulated HSP27 induction in osteoblasts. J Cell Biochem 2006, 98:1249-1256

35. Ruzinova MB, Benezra R: ID proteins in development, cell cycle and cancer. Trends Cell Biol 2003, 13:410-418.

36. Kim HJ, Chung H, Yoo YG, Kim H, Lee JY, Lee MO, Kong G: Inhibitor of DNA binding 1 activates vascular endothelial growth factor through enhancing the stability and activity of hypoxia-inducible factor-1A. Mol Cancer Res 2007, 5:321-329.

37. Ong LL, Lin PC, Zhang X, Chia SM, YU H: Kinectin-dependent assembly of translation elongation factor-1 complex on endoplasmic reticulum regulates protein synthesis. J Biol Chem 2006, 281:33621-33634.

38. Tamamura Y, Otani T, Kanatani N, Koyama E, Kitagaki J, Komori T, Yamada Y, Costantini F, Wakisaka S, Pacifici M, Iwamoto M, Enomoto-Iwamoto M: Developmental Regulation of Wnt/B-Catenin Signals Is Required for Growth Plate Assembly, Cartilage Integrity, and Endochondral Ossification. J Bio Chem 2005, 280:19185-19195.

39. Nakanishi R, Shimizu M, Mori M, Akiyama H, Okudaira S, Otsuki B, Hashimoto M, Higuchi K, Hosokawa M, Tsuboyama T, Nakamura1 T: Secreted frizzledrelated protein 4 is a negative regulator of peak BMD in SAMP6 Mice. J Bone Miner Res 2006, 21:1713-1721.

40. Horvath LG, Lelliott JE, Kench JG, Lee CS, Williams ED, Saunders DN, Grygie $\mathrm{JJ}$, Sutherland RL, Henshall SM: Secreted frizzled-related protein 4 inhibits proliferation and metastatic potential in prostate cancer. Prostate 2007, 67:1081-1090.

doi:10.1186/1471-2164-14-276

Cite this article as: Tian et al:: Screening of differentially expressed genes in the growth plate of broiler chickens with Tibial Dyschondroplasia by microarray analysis. BMC Genomics 2013 14:276.

\section{Submit your next manuscript to BioMed Central and take full advantage of:}

- Convenient online submission

- Thorough peer review

- No space constraints or color figure charges

- Immediate publication on acceptance

- Inclusion in PubMed, CAS, Scopus and Google Scholar

- Research which is freely available for redistribution

Submit your manuscript at www.biomedcentral.com/submit
C Biomed Central 